\title{
Down-regulation of annexin II in prostate cancer is associated with Gleason score, recurrence, metastasis and poor prognosis
}

\author{
TAO DING ${ }^{1,2}$, LUOYAN YANG $^{1}$, YINHUAI WANG ${ }^{1}$, JUNBIN YUAN $^{1}$, TIEDING CHEN $^{1}$ and XIAOJIAN CAI ${ }^{2}$ \\ ${ }^{1}$ Department of Urology, The Second Xiangya Hospital of Central South University, Changsha 410011; \\ ${ }^{2}$ Department of Urology, The First Affiliated Hospital, University of South China, Hengyang 421001, P.R. China
}

Received March 22, 2010; Accepted July 2, 2010

DOI: $10.3892 / \mathrm{mmr} .2010 .332$

\begin{abstract}
The aim of this study was to compare the expression of annexin II (ANXA2) in benign prostatic hyperplasia (BPH) with that of prostate cancer (PC), and to correlate the expression levels with pathologic grade and stage. Immunohistochemistry was performed on samples from 85 patients with PC and 40 patients with $\mathrm{BPH}$. The correlation between ANXA2 expression and clinicopathologic features and clinical outcome was evaluated. The data showed that ANXA2 expression was significantly lower in PC compared to $\mathrm{BPH}(\mathrm{P}<0.01)$. There was significant difference between ANXA2 expression and Gleason score $(\mathrm{P}<0.01)$. Patients with down-regulated ANXA2 tended to have tumors of advanced clinical stage, more frequent recurrence and regional lymph node and distant metastasis. ANXA2 expression was not correlated with age. The down-regulation of ANXA2 in a PC-3 cell line increased in vitro invasive ability, and ANXA2 had an independent prognostic effect on overall survival. In conclusion, ANXA2 dysregulation is an important event associated with the development and progression of PC. ANXA2 down-regulation aids in the discrimination of $\mathrm{PC}$ from $\mathrm{BPH}$ and may serve as a clinically useful biomarker.
\end{abstract}

\section{Introduction}

Annexins have roles in diverse cellular functions, including apoptosis, membrane trafficking, signal transduction, cellular motility and cell-cell interactions (1-3). Annexin II (ANXA2) is a member of a family of calcium-dependent phospholipid membrane-binding proteins that contains a conserved repeating domain of approximately 70 amino acids. ANXA2 has been shown to concentrate in lipid rafts and likely participates in ion channel regulation and the formation of tight junctions $(4,5)$. It is up-regulated in response to physiologic

Correspondence to: Dr Luoyan Yang, Department of Urology, The Second Xiangya Hospital of Central South University, Changsha 410011, P.R. China

E-mail: dyangluoyan@yahoo.com.cn

Key words: prostate cancer, annexin II, Gleason scores, prognosis stress, and inhibits cellular mobility when levels are restored in prostate cancer cells in culture. Several annexin family protein members are underexpressed in prostate cancer. The down-regulation of annexin I protein expression in prostate cancer was first demonstrated by molecular profiling studies of human prostate cancer samples (6). ANXA2 is also reduced in human prostate cancer, and biological studies in which ANXA2 expression was restored suggest that the dysregulation of ANXA2 expression may be an important early event in the initiation of prostate cancer $(7,8)$. Although reduced ANXA2 expression in human prostate cancer has previously been demonstrated, the biologic significance of the differential ANXA2 expression is not known. In the present study, we evaluated the expression of ANXA2 in prostate cancer, and examined its role by reducing ANXA2 expression levels in PC-3 cells using short hairpin RNA (shRNA) and analyzing the characteristics of malignancy. In addition, the levels of ANXA2 protein expression and the risk of recurrence were retrospectively evaluated in a small cohort. The results suggest a positive role for ANXA2 expression in highly aggressive prostate cancer, and demonstrate a specific role for ANXA2 in prostate cancer cells.

\section{Materials and methods}

Patients and specimens. Specimens were obtained from 85 patients with prostate cancer (PC) and 40 patients with benign prostatic hyperplasia $(\mathrm{BPH})$ admitted to The First Affiliated Hospital of the University of South University and the Second Xiangya Hospital of Central South University. The samples were collected during radical prostatectomy or via stab specimens. The mean age of the PC patients was 64.3 years (range 51-80). Specimens from the $40 \mathrm{BPH}$ patients served as the control.

Immunohistochemical staining. Immunohistochemistry was performed on formalin-fixed and paraffin-embedded tissue sections using a standard immunohistochemical technique. The specimens were fixed in $10 \%$ formalin, embedded in paraffin and cut into 3- to 5- $\mu$ m-thick sections. Tissues were deparaffinized and rehydrated through a graded series of ethanol. After blocking of endogenous peroxidase activity with $0.75 \%$ hydrogen peroxide in methanol for $45 \mathrm{~min}$, the sections were microwaved for $5 \mathrm{~min}$ twice at maximum 
power in $10 \mathrm{mM}$ citrate buffer to enhance antigen detection, then cooled in buffer for $20 \mathrm{~min}$ at room temperature. After incubation for $20 \mathrm{~min}$ with $5 \%$ normal horse serum diluted in phosphate-buffered saline (PBS), the sections were incubated for $1 \mathrm{~h}$ at room temperature with a 1:200 dilution of mouse ANXA2 antibody. After washing in PBS buffer, the secondary absorbed horse anti-mouse immunoglobulins in the 1:200 dilution and the reaction product were visualized with $0.03 \%$ hydrogen peroxide as the substrate and diaminobenzidine as a chromogen. The sections were counterstained with hematoxylin and mounted on mounting medium. As the negative control, prostatic tissue from the same patient, processed as above except for being incubated with only the diluent without the ANXA2 antibody, was used. Normal prostatic tissue served as the positive control.

Evaluation of staining. Sections were evaluated using light microscopy (Olympus) by two blinded investigators to provide a consensus on the staining pattern. Each cancerous region was assigned a Gleason score according to the staining intensity of the cancerous region. At least 10 high-power fields were selected at random and $>1,000$ cells were counted in each section. The staining intensity was graded as: 0 , no staining; 1+, mild staining; 2+, moderate staining; and 3+, intense staining. The area of staining was evaluated as: 0 , no staining of cells in any microscopic fields; $1+,<30 \%$ of tissue stained positive; $2+, 30-60 \%$ stained positive; and $3+,>60 \%$ stained positive. The scores (extension + intensity) were then summed, for a minimum score of 0 and a maximum of 6 . A combined staining score (extension + intensity) of 2 indicated negative (low) staining; a score of 3-4, moderate staining; and a score of 5-6, strong staining.

Western blotting. Briefly, $40 \mu \mathrm{g}$ of lysates were separated by $10 \%$ SDS-PAGE and transferred to a polyvinylidene difluoride membrane. Blots were blocked with $5 \%$ non-fat dry milk for $2 \mathrm{~h}$ at room temperature and then incubated with primary anti-ANXA2 antibody for $2 \mathrm{~h}$ at room temperature, followed by incubation with a horseradish peroxidase-conjugated secondary antibody for $1 \mathrm{~h}$ at room temperature. The signal was visualized with an enhanced chemiluminescence detection reagent and quantitated by densitometry using the ImageQuant image analysis system (Storm Optical Scanner; Molecular Dynamics, Sunnyvale, CA, USA). The mouse antiactin antibody (1:5,000; Sigma) was detected simultaneously as a loading control.

Administration of ANXA2 siRNA to PC-3 cells. PC-3 prostate cancer cells were transfected with ANXA2 siRNA or control siRNA (Santa Cruz Biotechnology) according to the siRNA transfection protocol provided by the manufacturer. Briefly, the day before transfection, PC-3 cells were plated onto 6-well plates at a density of $10^{5}$ cells/ml in RPMI-1640 medium (Invitrogen, Carlsbad, CA, USA) containing 10\% FBS. When the cells had reached $60-80 \%$ confluency, they were transfected with $10 \mathrm{nmol} / \mathrm{l}$ of ANXA2 siRNA or control siRNA after pre-incubation for 20 min with siRNA transfection reagent in siRNA transfection medium. The medium was replaced with in RPMI-1640 medium containing 10\% FBS $4 \mathrm{~h}$ after the initiation of the transfection, and the cell culture was continued for an additional $44 \mathrm{~h}$. At the end of the transfection, ANXA2 expression levels in the cells were determined by Western blotting.

In vitro cell invasion assay. The invasiveness of the PC-3 cells transfected with ANXA2 siRNA, Lipofectamine 2000 (Lip) or control siRNA was evaluated in 24-well transwell chambers (Costar, Cambridge, MA, USA) as directed by the manufacturer. Briefly, the upper and lower culture compartments of each well were separated by polycarbonate membranes ( $8 \mu \mathrm{m}$ pore size). The membranes were pre-coated with $100 \mu \mathrm{g} / \mathrm{cm}^{2}$ of collagen matrix (Matrigel; Collaborative Biomedical Products, Bedford, MA, USA), which was reconstituted by the addition of $0.5 \mathrm{ml}$ of serum-free RPMI1640 medium to the well for $2 \mathrm{~h}$. To assess the ability of the cells to penetrate the pre-coated polycarbonate membrane, $1.25 \times 10^{4}$ cells in $0.5 \mathrm{ml}$ of RPMI-1640 medium containing $1 \%$ FBS was placed into the upper compartment of the wells, and $0.75 \mathrm{ml}$ of RPMI-1640 medium containing $10 \%$ FBS was placed into the lower compartment. The transwell chambers were incubated for $24 \mathrm{~h}$ at $37^{\circ} \mathrm{C}$ in a humidified $5 \% \mathrm{CO}_{2}$ atmosphere. Invaded cells attached underneath the chamber membrane were stained with a Diff-Quik stain kit (Dade Behring, Newark, DE, USA) and counted in eight random fields with an inverted microscope at a magnification of x200. Invasive ability was defined as the average number of cell to penetrated the matrix-coated membrane per field. The experiment was performed in triplicate.

Statistical analysis. Statistical analyses were performed using SPSS software (version 17.0) . Significant differences between ANXA2 expression in the PC tumor and BPH samples were censored, and the missing values were replaced by the series mean method. A univariate analysis with the Cox proportional hazards model was used to determine each identified prognostic factor, while multivariate analysis with the Cox proportional hazards model was used to explore the combined effects. Significant differences between the expression of ANXA2 and clinical variables including age, Gleason score, tumor stage, lymph node metastasis, distant metastasis, clinical stage and recurrence were compared using the non-parametric Kruskal-Wallis test and a pairwise Mann-Whitney U test. Survival rates (cumulative survival and cumulative survival curves) were obtained by the Kaplan-Meier method and differences between the curves were compared by log-rank analysis. The level of statistical significance was set at a P-value of $<0.05$.

\section{Results}

Expression of ANXA2 in clinical samples. The expression of ANXA2 was detected using immunohistochemistry in formalin-fixed and paraffin-embedded archival clinical tissues from 85 cases of PC and 40 cases of BPH. As shown in Fig. 1 and Table I, the expression level of ANXA2 was significantly lower in the $\mathrm{PC}$ compared to the $\mathrm{BPH}$ specimens $(\mathrm{P}<0.01)$. There was significant difference between the expression of ANXA2 and Gleason score (Table II, P<0.01). The results indicated that the down-regulation of ANXA2 protein might be related to $\mathrm{PC}$ development. 

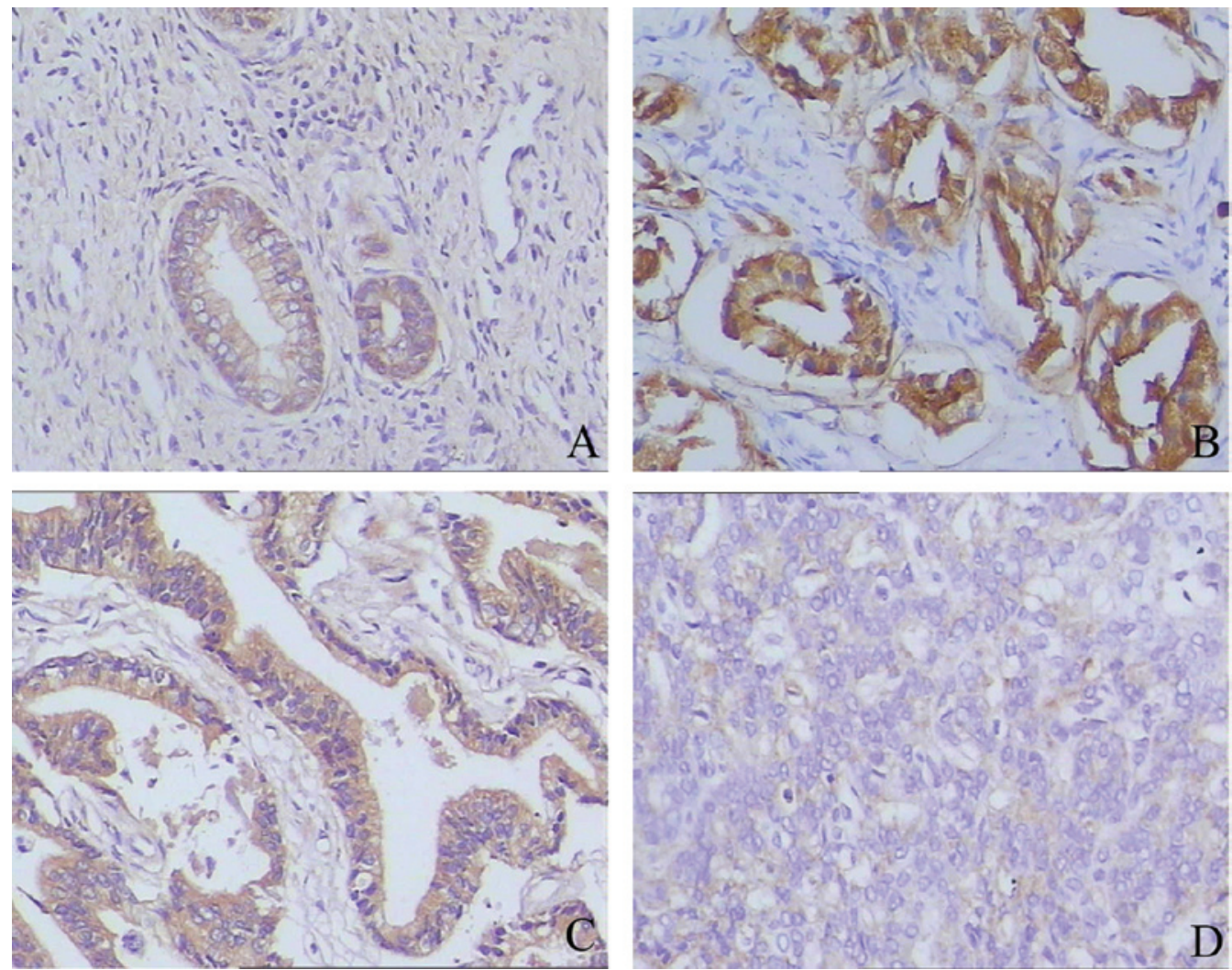

Figure 1. Expression of ANXA2 in prostate cancer (PC) and benign prostatic hyperplasia (BPH) specimens. (A) BPH; (B) well-differentiated PC; (C) moderately-differentiated PC; (D) poorly-differentiated PC. Magnification x200.

Table I. Expression of ANXA2 in benign prostatic hyperplasia (BPH) and prostate cancer (PC).

\begin{tabular}{lccccr}
\hline & No. & \multicolumn{4}{c}{ Staining } \\
\cline { 3 - 6 } & & Low (0-2) & Moderate (3-4) & Strong (5-6) & P-value \\
\hline BPH & 40 & 3 & 12 & 25 & 0.000 \\
PC & 85 & 32 & 48 & 5 & \\
\hline
\end{tabular}

$\mathrm{P}<0.05$, Mann-Whitney U test.

Table II. Relationship between ANXA2 expression and Gleason score.

\begin{tabular}{lccccr}
\hline & No. & \multicolumn{4}{c}{ Staining } \\
\cline { 3 - 6 } & & Low (0-2) & Moderate (3-4) & Strong (5-6) & P-value \\
\hline Gleason score & & 9 & 27 & 4 & 0.001 \\
$2-4$ & 40 & 15 & 20 & 1 & \\
$5-7$ & 36 & 8 & 1 & 0 & \\
$8-10$ & 9 & & & & \\
\end{tabular}

$\mathrm{P}<0.05$, Kruskal-Wallis test.

Relationship between ANXA2 expression and clinicopathologic features. Table II shows the association of several clinicopathologic factors with ANXA2 expression in 85 cases of PC.
ANXA2 expression was correlated with tumor stage, Gleason score, recurrence, lymph node metastasis and distant metastasis $(\mathrm{P}<0.05)($ Table III). Tumors exhibiting down-regulated 
Table III. Relationship between ANXA2 expression and clinicopathologic features.

\begin{tabular}{|c|c|c|c|c|c|}
\hline & \multirow[t]{2}{*}{ No. } & \multicolumn{4}{|c|}{ Staining } \\
\hline & & Low (0-2) & Moderate (3-4) & Strong (5-6) & P-value \\
\hline Age (years) & & & & & 0.201 \\
\hline$\geq 60$ & 50 & 22 & 25 & 3 & \\
\hline$<60$ & 35 & 10 & 23 & 2 & \\
\hline Lymph node metastasis & & & & & $0.000^{\mathrm{a}}$ \\
\hline Yes & 33 & 26 & 6 & 0 & \\
\hline No & 52 & 5 & 42 & 5 & \\
\hline Distant metastasis & & & & & $0.000^{\mathrm{a}}$ \\
\hline Yes & 21 & 18 & 3 & 0 & \\
\hline No & 64 & 14 & 45 & 5 & \\
\hline Tumor stage & & & & & $0.000^{\mathrm{a}}$ \\
\hline $\mathrm{T} 1$ & 8 & & & & \\
\hline $\mathrm{T} 2$ & 25 & 4 & 26 & 4 & \\
\hline $\mathrm{T} 3$ & 29 & 28 & 22 & 1 & \\
\hline $\mathrm{T} 4$ & 23 & & & & \\
\hline Gleason scores & & & & & $0.001^{\mathrm{a}}$ \\
\hline $2-4$ & 40 & 9 & 27 & 4 & \\
\hline $5-7$ & 36 & 15 & 20 & 1 & \\
\hline $8-10$ & 9 & 8 & 1 & 0 & \\
\hline Recurrence & & & & & $0.000^{\mathrm{a}}$ \\
\hline Yes & 28 & 20 & 6 & 2 & \\
\hline No & 57 & 12 & 42 & 3 & \\
\hline
\end{tabular}

${ }^{a} \mathrm{P}<0.05$, Mann-Whitney U test or ANOVA.

Table IV. Prognostic factors according to univariate analysis (Cox proportional hazards model).

\begin{tabular}{lcr}
\hline Variables & Hazard ratio $(95 \%$ CI & P-value \\
\hline Age $(\geq 60 /<60)$ & $0.181(0.018-1.325)$ & 0.399 \\
Tumor stage (T1-T2/T3-T4) & $-0.764(0.434-2.082)$ & $0.012^{\mathrm{a}}$ \\
Gleason scores (2-4/5-7/8-10) & $-0.982(0.559-1.602)$ & $0.008^{\mathrm{a}}$ \\
Lymph node metastasis (yes/no) & $-0.527(0.197-1.552)$ & $0.002^{\mathrm{a}}$ \\
Distant metastasis (yes/no) & $-0.791(0.406-1.731)$ & $0.002^{\mathrm{a}}$ \\
Recurrence (yes/no) & $-1.412(1.056-5.134)$ & $0.010^{\mathrm{a}}$ \\
ANXA2 (0-2/3-4/5-6) & $3.113(1.954-9.886)$ & $0.000^{\mathrm{a}}$ \\
\hline
\end{tabular}

95\% CI, $95 \%$ confidence interval. ${ }^{\mathrm{P}}<0.05$.

Table V. Prognostic fators according to multivariate analysis (Cox proportional hazards model).

\begin{tabular}{lcr}
\hline Variables & $\begin{array}{c}\text { Hazard ratio } \\
(95 \% \text { confidence interval) }\end{array}$ & P-value \\
\hline Tumor stage (T2/T3) & $-0.341(0.132-1.073)$ & 0.210 \\
Gleason scores (2-4/5-10) & $-0.446(0.212-2.111)$ & $0.009^{\text {a }}$ \\
Lymph node metastasis (Yes/No) & $-0.594(0.302-3.114)$ & $0.005^{\text {a }}$ \\
Distant metastasis (Yes/No) & $-1.486(1.001-7.133)$ & $0.007^{\text {a }}$ \\
Recurrence (Yes/No) & $2.152(1.775-9.399)$ & $0.003^{\text {a }}$ \\
ANXA2 (0-2/3-6) & $0.000^{\text {a }}$
\end{tabular}

$\mathrm{a}<<0.05$. 



Figure 2. In vitro cell invasion assay of ANXA2 siRNA-transfected and control PC-3 cells. (A) Western blotting showing the expression level of ANXA2 in ANXA2 siRNA-transfected and control cells. (B) Invasion of ANXA2 siRNA-transfected and control cells, measured using transwell chambers. (C) Number of invasive tumor cells per field in ANXA2 siRNAtransfected and control PC-3 cells. Lip, lipofectamine groups.

ANXA2 tended to have advanced clinical stage, more frequent recurrence and regional lymph node and distant metastasis. The expression level of ANXA2 was not correlated with age.

Effect of ANXA2 down-regulation by siRNA on cell invasiveness of PC-3 cells. To study the association of ANXA2 down-regulation in the PC-3 cell line with its metastatic ability, PC-3 cells were transfected with ANXA2 siRNA, then the siRNA-induced inhibition of ANXA2 expression was determined by Western blotting. As shown in Fig. 2A, the introduction of ANXA2 siRNA into PC-3 cells significantly decreased ANXA2 expression, whereas ANXA2 expression was not significantly suppressed by control siRNA. Next, the effect of ANXA2 down-regulation by siRNA on the cell invasiveness of the PC- 3 cell line was evaluated using an in vitro invasion assay. As shown in Fig. $2 \mathrm{~B}$ and $\mathrm{C}, \mathrm{PC}-3$ cells transfected with ANXA2 siRNA were approximately 3.5-fold less invasive than PC-3 cells transfected with control siRNA $(\mathrm{P}<0.01)$, indicating that the down-regulation of ANXA2 expression increased the in vitro invasive ability of the PC-3 cell line. ANXA2 down-regulation was thus positively associated with the invasiveness of the PC-3 cells in vitro.

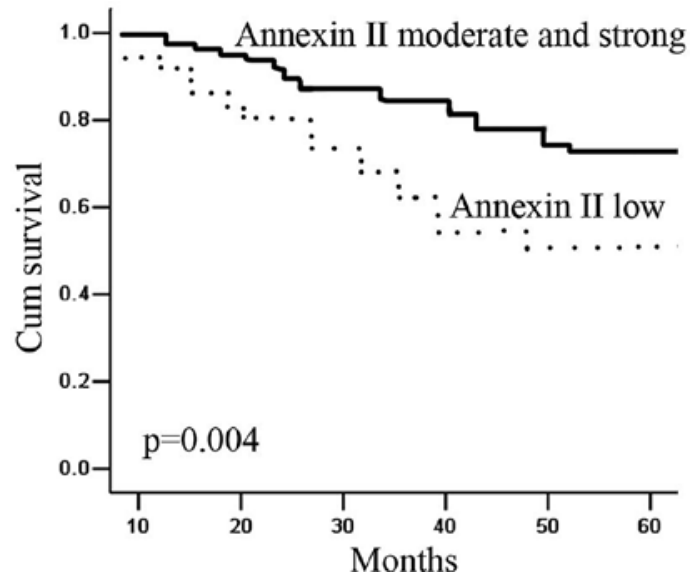

Figure 3. Kaplan-Meier survival curve of the expression of ANXA2 in prostate cancer $(\mathrm{P}=0.004)$.

Survival analysis. Survival curves were calculated for the 85 patients with PC. One patient (1.2\%) was lost during follow-up. By the end of the study, 24 patients (28.2\%) had succumbed to PC, 60 patients $(70.6 \%)$ were still alive, 28 patients $(32.9 \%)$ had experienced disease recurrence, and 21 patients (24.7\%) had presented distant metastasis. The mean follow-up time was $57.8 \pm 11.7$ months. Using univariate analysis (Cox proportional hazards model), the following variables were found to be significantly associated with prognosis: tumor stage, Gleason score, recurrence, lymph node metastasis, distant metastasis and the expression level of ANXA2 $(\mathrm{P}<0.05$, Table IV). Survival curves calculated by the Kaplan-Meier method and analyzed using the log-rank test showed that the survival rates of the patients were significantly related with the down-regulation of ANXA2 ( $<<0.01$, Fig. 3). Multivariate analysis showed that Gleason score, recurrence, distant metastasis and the expression level of ANXA2 had an independent prognostic effect on overall survival $(\mathrm{P}<0.01$, Table $\mathrm{V})$.

\section{Discussion}

Annexins constitute a family of $\mathrm{Ca}^{2+}$-dependent membranebinding proteins, of which at least 20 have been described to date. Among these, ANXA2 has been revealed to be a multifunctional protein in vitro; however, its role in vivo requires further investigation. ANXA2 plays a key role in numerous cellular functions, including apoptosis, signal transduction and cellular motility (9-11). Increased expression of ANXA2 has been reported in various types of cancer, including lung cancer, hepatocellular carcinoma, acute myelogenous leukemia, clear-cell renal cell carcinoma and breast cancer (12-16). Several studies have also demonstrated the downregulation of ANXA2 expression in PC $(8,17)$. Banerjee et al (18) compared the expression of ANXA2 in patients with PC from India and the United States, and observed focal expression of ANXA2 in the patients with poorly-differentiated PC from the United States, but not in those from India. These results are in contrast to the findings of present study, which indicate that ANXA2 was absent or focally present in patients with poor differentiation, but was retained in patients with high-grade PC. 
Although ANXA2 is commonly down-regulated in PC, the association between the reduced expression of ANXA2 and pathologic grade and stage is unknown (19), and the mechanisms affecting ANXA2 expression levels in PC remain to be determined. In this study, the expression level of ANXA2 was significantly lower in PC compared to BPH specimens. There was a significant difference between ANXA2 expression and Gleason score, and expression levels were higher in well- or moderately-differentiated compared to poorlydifferentiated prostate adenocarcinoma. The results indicated that the down-regulation of ANXA2 protein may be related to the development and differentiation of PC. ANXA2 expression was correlated with tumor stage, Gleason score, recurrence, lymph node metastasis and distant metastasis. Tumors exhibiting down-regulated ANXA2 tended to have advanced clinical stage, more frequent recurrence and regional lymph node and distant metastasis. ANXA2 expression was not correlated with age. Regardless of the mechanisms behind reduced ANXA2 expression in PC, the data suggest that ANXA2 may be a molecular determinant of more aggressive PC, and serves as an independent prognostic indicator of overall survival.

ANXA2 expression also correlates with Fuhrman nuclear grade and clinical outcome in conventional renal cell carcinoma (20). In brain tumors, ANXA2 is expressed in glioblastoma multiforme and in anaplastic astrocytoma, but not in lower-grade tumors (21). In vitro studies on pancreatic adenocarcinoma cells have shown that ANXA2 is a binding site for tissue-type plasminogen activator (t-PA) (22). Active $\mathrm{t}$-PA at the cell surface of pancreatic adenocarcinoma causes local activation of plasmin, which increases the invasiveness of tumor cells (23). This has also been observed in transgenic mouse models of pancreatic cancer (24). ANXA2 has a binding site for cathepsin $\mathrm{B}$ and t-PA, and reacts with extracellular surface proteins. Increased expression of ANXA2 leads to increased invasion and motility (25). However, decreased expression of ANXA2 in poorly-differentiated PC results in the increased ability of the cancers to invade locally. Moreover, ANXA2 expression is lost or reduced in PC cells, and its re-expression inhibits PC cell migration (19). Since ANXA2 likely plays a role in cell-cell interaction and tight junction formation, it is possible that the down-regulation of ANXA2 in PC is an attempt by the carcinoma to increase PC cell migration and therefore metastasis.

In order to determine whether ANXA2 contributes to the migration and metastasis of PC, we established stable cell lines deriving from PC-3 cells in which endogenous ANXA2 expression was inhibited by shRNA. The results indicated that the down-regulation of ANXA2 expression in the PC-3 cell line increased its in vitro invasive ability; thus, ANXA2 down-regulation was positively associated with the invasiveness of the PC-3 cell line in vitro. Shiozawa et al (26) also found that PC cell lines migrate towards ANXA2, and that the adhesion of PC cells to osteoblasts and endothelial cells is possibly inhibited by ANXA2. The mechanisms of metastasis may involve in the adhesion and localization of PC. The data showed that ANXA2 down-regulation may aid in the discrimination of PC from BPH and that ANXA2 may serve as a clinically useful biomarker.

In conclusion, ANXA2 expression is commonly downregulated in prostate cancer, suggesting that ANXA2 dysregulation is an important event associated with prostate cancer development and progress and plays a central role in prostate cancer metastasis.

\section{References}

1. Ang EZ, Nguyen HT, Sim HL, Putti TC and Lim LH: Annexin-1 regulates growth arrest induced by high levels of estrogen in MCF-7 breast cancer cells. Mol Cancer Res 7: 266-274, 2009.

2. Mussunoor S and Murray GI: The role of annexins in tumour development and progression. J Pathol 216: 131-140, 2008.

3. Nakahara S and Raz A: Biological modulation by lectins and their ligands in tumorprogression and metastasis. Anticancer Agents Med Chem 8: 22-36, 2008.

4. Zhang F, Zhang L, Zhang B, Wei X, Yang Y, Qi RZ, Ying G, Zhang $\mathrm{N}$ and Niu R: Anxa2 plays a critical role in enhanced invasiveness of the multidrug resistant human breast cancer cells. J Proteome Res 8: 5041-5047, 2009.

5. Bao H, Jiang M, Zhu M, Sheng F, Ruan J and Ruan C: Overexpression of Annexin II affects the proliferation, apoptosis, invasion and production of proangiogenic factors in multiple myeloma. Int J Hematol 90: 177-185, 2009.

6. Inokuchi J, Lau A, Tyson DR and Ornstein DK: Loss of annexin A1 disrupts normal prostate glandular structure by inducing autocrine IL-6 signaling. Carcinogenesis 30: 1082-1098, 2009.

7. Liu J and Vishwanatha JK: Regulation of nucleo-cytoplasmic shuttling of human annexin A2: a proposed mechanism. Mol Cell Biochem 303: 211-220, 2007.

8. Inokuchi J, Narula N, Yee DS, Skarecky DW, Lau A, Ornstein DK and Tyson DR: Annexin A2 positively contributes to the malignant phenotype and secretion of IL-6 in DU145 prostate cancer cells. Int J Cancer 124: 68-74, 2009.

9. Kido O, Fukushima K, Ueno Y, Inoue J, Jefferson DM and Shimosegawa T: Compensatory role of inducible annexin A2 for impaired biliary epithelial anion-exchange activity of inflammatory cholangiopathy. Lab Invest 89: 1374-1386, 2009.

10. Nedjadi T, Kitteringham N, Campbell F, Jenkins RE, Park BK, Navarro P, Ashcroft F, Tepikin A, Neoptolemos JP and Costello E: S100A6 binds to annexin 2 in pancreatic cancer cells and promotes pancreatic cancer cell motility. Br J Cancer 101: 1145-1154, 2009.

11. Grewal T and Enrich C: Annexins - modulators of EGF receptor signalling and trafficking. Cell Signal 21: 847-858, 2009.

12. Ji NY, Park MY, Kang YH, Lee CI, Kim DG, Yeom YI, Jang YJ, Myung PK, Kim JW, Lee HG, Kim JW, Lee K and Song EY: Evaluation of annexin II as a potential serum marker for hepatocellular carcinoma using a developed sandwich ELISA method. Int J Mol Med 24: 765-771, 2009.

13. Mohammad HS, Kurokohchi K, Yoneyama H, et al: Annexin A2 expression and phosphorylation are up-regulated in hepatocellular carcinoma. Int J Oncol 33: 1157-1163, 2008.

14. Huang Y, Jin Y, Yan CH, Yu Y, Bai J, Chen F, Zhao YZ and Fu SB: Involvement of Annexin A2 in p53 induced apoptosis in lung cancer. Mol Cell Biochem 309: 117-123, 2008.

15. Park MH, Cho SA, Yoo KH, Yang MH, Ahn JY, Lee HS, Lee KE, Mun YC, Cho DH, Seong CM and Park JH: Gene expression profile related to prognosis of acute myeloid leukemia. Oncol Rep 18: 1395-1402, 2007.

16. Rodrigo Tapia JP, Pena Alonso E, García-Pedrero JM, Florentino Fresno M, Suárez Nieto C, Owen Morgan R and Fernández MP: Annexin A2 expression in head and neck squamous cell carcinoma. Acta Otorrinolaringol Esp 58: 257-262, 2007.

17. Ellinger J, Bastian PJ, Jurgan T, Biermann K, Kahl P, Heukamp LC, Wernert N, Müller SC and von Ruecker A: CpG island hypermethylation at multiple gene sites in diagnosis and prognosis of prostate cancer. Urology 71: 161-167, 2008.

18. Banerjee AG, Liu J, Yuan Y, Gopalakrishnan VK, Johansson SL, Dinda AK, Gupta NP, Trevino L and Vishwanatha JK: Expression of biomarkers modulating prostate cancer angiogenesis: differential expression of annexin II in prostate carcinomas from India and USA. Mol Cancer 2: 34, 2003.

19. Yee DS, Narula N, Ramzy I, Boker J, Ahlering TE, Skarecky DW and Ornstein DK: Reduced annexin II protein expression in high-grade prostatic intraepithelial neoplasia and prostate cancer. Arch Pathol Lab Med 131: 902-908, 2007.

20. Zimmermann U, Woenckhaus C, Pietschmann S, Junker H, Maile S, Schultz K, Protzel C and Giebel J: Expression of annexin II in conventional renal cell carcinoma is correlated with Fuhrman grade and clinical outcome. Virchows Arch 445: 368-374, 2004. 
21. Reeves SA, Chavez-Kappel C, Davis R, Rosenblum M and Israel MA: Developmental regulation of annexin II (lipocortin 2) in human brain and expression in high grade glioma. Cancer Res 52: 6871-6876, 1992.

22. Diaz VM, Hurtado M, Thomson TM, Reventos J and Paciucci R: Specific interaction of tissue-type plasminogen activator (t-PA) with annexin II on the membrane of pancreatic cancer cells activates plasminogen and promotes invasion in vitro. Gut 53 : 993-1000, 2004

23. Paciucci R, Tora M, Diaz VM and Real FX: The plasminogen activator system in pancreas cancer: role of t-PA in the invasive potential in vitro. Oncogene 16: 625-633, 1998.
24. Aguilar S, Corominas JM, Malats N, Pereira JA, Dufresne M, Real FX and Navarro P: Tissue plasminogen activator in murine exocrine pancreas cancer. Am J Pathol 165: 1129-1139, 2004.

25. Mai J, Waisman DM and Sloane BR: Cell surface complex of cathepsin B/annexin II tetramer in malignant progression. Biochem Biophys Acta 1477: 215-230, 2000.

26. Shiozawa Y, Havens AM, Jung Y, Ziegler AM, Pedersen EA, Wang J, Wang J, Lu G, Roodman GD, Loberg RD, Pienta KJ and Taichman RS: Annexin II/annexin II receptor axis regulates adhesion, migration, homing, and growth of prostate cancer. $\mathrm{J}$ Cell Biochem 105: 370-380, 2008. 